\title{
A NOTE ON $P_{1}$ - AND LIPSCHITZIAN MATRICES*
}

\author{
G. S. R. MURTHY ${ }^{\dagger}$, S. K. NEOGY $\ddagger$, AND F. THUIJSMAN§
}

\begin{abstract}
The linear complementarity problem $(q, A)$ with data $A \in R^{n \times n}$ and $q \in R^{n}$ involves finding a nonnegative $z \in R^{n}$ such that $A z+q \geq 0$ and $z^{t}(A z+q)=0$. Cottle and Stone introduced the class of $P_{1}$-matrices and showed that if $A$ is in $P_{1} \backslash Q$, then $K(A)$ (the set of all $q$ for which $(q, A)$ has a solution) is a half-space and $(q, A)$ has a unique solution for every $q$ in the interior of $K(A)$. Extending the results of Murthy, Parthasarathy, and Sriparna [Ann. Dynamic Games, to appear], we present a number of equivalent characterizations of $P_{1} \backslash Q$. Also, we present yet another characterization of $P$-matrices. This widens the range of matrix classes for which a conjecture raised by Murthy, Parthasarathy, and Sriparna [SIAM J. Matrix Anal. Appl., 19 (1998), pp. 898-905] characterizing the class of Lipschitzian matrices is true.
\end{abstract}

Key words. linear complementarity problem, matrix classes, Lipschitz property

AMS subject classification. $90 \mathrm{C} 33$

PII. S0895479897329898

1. Introduction. The linear complementarity problem (LCP) with data $A \in$ $\boldsymbol{R}^{n \times n}$ and $q \in \boldsymbol{R}^{n}$ involves finding a vector $z \in \boldsymbol{R}^{n}$ such that

$$
A z+q \geq 0, \quad z \geq 0, \quad \text { and } z^{t}(A z+q)=0 .
$$

Many have tackled a number of problems on existence and solvability of LCP by studying the properties of $A$; this has lead to the evolution of a wide range of matrix classes. Good background material on the subject can be found in $[2,13]$.

We shall briefly introduce the concepts and notation required for presentation of the results of this paper. For $A \in \boldsymbol{R}^{n \times n}, q \in \boldsymbol{R}^{n}$, let $F(q, A)=\left\{z \in \boldsymbol{R}_{+}^{n}: A z+q \geq 0\right\}$, $S(q, A)=\left\{z \in F(q, A):(A z+q)^{t} z=0\right\}$, and $K(A)=\left\{p \in \boldsymbol{R}^{n}: S(p, A) \neq \emptyset\right\}$. For most of the other notation, we shall follow that used in [2]. Let $\bar{n}=\{1,2, \ldots, n\}$ and for $\alpha, \beta \subseteq \bar{n}$, let $A_{\alpha \beta}$ denote the submatrix of $A$ obtained by retaining only rows corresponding to $\alpha$ and columns corresponding to $\beta$. A similar notation is used for vectors. The complement of $\alpha \subseteq \bar{n}$ with respect to $\bar{n}$ will be denoted by $\bar{\alpha}$. The vector $e$ will denote the vector of ones (of appropriate order).

We define a number of matrix classes studied extensively in the literature of LCPs. Say that $A \in \boldsymbol{Q}$ if $S(q, A) \neq \phi$ for all $q \in \boldsymbol{R}^{n} ; A \in \boldsymbol{Q}_{0}$ if for all $q, F(q, A) \neq \phi \Rightarrow$ $S(q, A) \neq \phi ; A \in \boldsymbol{P}_{0}(\boldsymbol{P})$ ( $A$ is nondegenerate) if $\operatorname{det} A_{\alpha \alpha} \geq 0(>0)(\neq 0)$ for all $\alpha \subseteq \bar{n}$; $A \in \boldsymbol{P}_{1}$ if $A \in \boldsymbol{P}_{0}$ and $\operatorname{det} A_{\alpha \alpha}=0$ for some unique $\alpha ; A \in \mathbf{I N} \boldsymbol{S}_{k}$ if $|S(q, A)|=k$ for all $q \in \operatorname{int} K(A) ; A \in \boldsymbol{R}_{0}$ if $|S(0, A)|=1 ; A \in \boldsymbol{E}_{0}(\boldsymbol{E})$ if $|S(q, A)|=1$ for all $q>0(q \geq 0)$; and $A \in \boldsymbol{E}^{\prime}$ if $|S(q, A)|=1$ for all $q \geq 0$ and $|S(0, A)| \geq 2$. The class

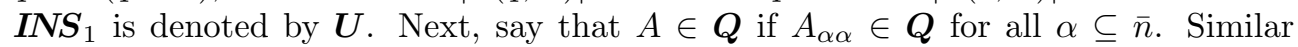
notation is used for other classes.

For $A \in \boldsymbol{R}^{n \times n}$ with $\operatorname{det} A_{\alpha \alpha} \neq 0$ for an $\alpha$, the principal pivotal transform (PPT) of $A$ with respect to $\alpha$, denoted by $\wp_{\alpha}(A)$, is defined as $M=\wp_{\alpha}(A)$, where $M_{\alpha \alpha}=$

*Received by the editors November 10, 1997; accepted for publication (in revised form) by R. Cottle April 6, 1998; published electronically December 28, 1999.

http://www.siam.org/journals/simax/21-2/32989.html

†Indian Statistical Institute, Street No. 8, Habsiguda, Hyderabad 500 007, India (murthygsr@ hotmail.com).

${ }_{\ddagger}^{\ddagger}$ Indian Statistical Institute, 7 SJS Sansanwal Marg, New Delhi 110 016, India (skn@isid.ac.in).

$\S$ University of Maastricht, P.O. Box 616,6200 MD Maastricht, The Netherlands (frank@math. unimaas.nl). 
$\left(A_{\alpha \alpha}\right)^{-1}, M_{\alpha \bar{\alpha}}=-\left(A_{\alpha \alpha}\right)^{-1} A_{\alpha \bar{\alpha}}, M_{\bar{\alpha} \alpha}=A_{\bar{\alpha} \alpha}\left(A_{\alpha \alpha}\right)^{-1}$, and $M_{\bar{\alpha} \bar{\alpha}}=A_{\bar{\alpha} \bar{\alpha}}-M_{\bar{\alpha} \alpha} A_{\alpha \bar{\alpha}}$. Whenever we refer to PPTs, we mean only those which are well defined. It is a wellestablished result that if $A$ belongs to $\boldsymbol{Q}, \boldsymbol{Q}_{0}, \boldsymbol{P}_{0}, \boldsymbol{P}, \boldsymbol{P}_{1}$, or $\boldsymbol{I N} \boldsymbol{S}_{k}$, then every PPT of $A$ is also in the respective class.

For any $A \in \boldsymbol{R}^{m \times n}$, the associated two-person zero-sum matrix game is described as follows. There are two players: row player $R$ and column player $C$. $R$ chooses a row $i, C$ chooses a column $j$, and $R$ pays $C$ an amount equal to $a_{i j}$. A mixed strategy for $R(C)$ is a probability vector $p \in \boldsymbol{R}^{m}\left(q \in \boldsymbol{R}^{n}\right)$. The fundamental theorem of von Neumann asserts that there exist strategies $\bar{p}$ and $\bar{q}$, called optimal strategies for $R$ and $C$, respectively, and a real number $v$, called the value of the game (denoted by $v(A)$ ), such that

$$
\bar{p}^{t} A \leq v e \text { and } A \bar{q} \geq v e .
$$

A strategy is said to be completely mixed if all its coordinates are positive. A game is said to be completely mixed if all the optimal strategies are completely mixed. We shall refer to the game by the matrix itself.

Cottle and Stone [3] showed that if $A \in \boldsymbol{P}_{1} \backslash \boldsymbol{Q}$, then $A \in \boldsymbol{U}$ and that $K(A)$ is a half-space. They further showed that if, in addition, $\operatorname{det} A=0$, then the support of the half-space $K(A)$ can be chosen to be a positive vector. Murthy, Parthasarathy, and Sriparna [11] showed that if $A$ is a completely mixed game with $v(A)=0$, then $A \in \boldsymbol{P}_{1} \backslash \boldsymbol{Q}$ if and only if $A \in \boldsymbol{U}$. In section 2, we show that $A \in \boldsymbol{P}_{1} \backslash \boldsymbol{Q}$ if and only if $A \in \boldsymbol{U}$ and $K(A)$ is a half-space with a positive support. Indeed, we give a number of equivalent conditions of the statement that $A \in \boldsymbol{P}_{1} \backslash \boldsymbol{Q}$ and $\operatorname{det} A=0$.

In section 3, we provide some results relating to the conjecture raised by Murthy, Parthasarathy, and Sriparna [12] concerning a constructive characterization of Lipschitzian matrices. In particular, we show that a certain (constructive) property is necessarily satisfied by Lipschitzian matrices as well as nondegenerate $\boldsymbol{I N} \boldsymbol{S}$-matrices and that the converse is true with an additional assumption.

2. Results on $\boldsymbol{P}_{\mathbf{1}}$-matrices. For the sake of completeness, we state relevant portions of some known theorems.

Theorem 1 (see Cottle and Stone [3]). Suppose $A \in \boldsymbol{R}^{n \times n} \cap \boldsymbol{P}_{1}$. The following statements hold:

(i) There exists a unique $\alpha$ such that $\wp_{\alpha}(A) \in \boldsymbol{P}_{1}$ and $\operatorname{det} \wp_{\alpha}(A)=0$.

(ii) $A$ is in $\boldsymbol{Q}_{0}$.

(iii) If $A \notin \boldsymbol{Q}$ and $\operatorname{det} A=0$, then $A \in \boldsymbol{U}$ and $K(A)=\left\{q: \pi^{t} q \geq 0\right\}$ for some $\pi>0$.

TheOREm 2 (see Kaplansky [7]). Suppose $A \in \boldsymbol{R}^{m \times n}$. The following statements hold for the matrix game $A$ :

(i) If the row player $R$ has a completely mixed strategy, then $A q=v(A)$ e for all optimal strategies $q$ of the column player $C$.

(ii) If $v(A)=0$, then the game is completely mixed if and only if $[m=n$, $\operatorname{rank}(A)=n-1$, and all cofactors of $A$ are different from zero and have the same sign].

(iii) If the matrix game $A$ is completely mixed, then $v(A)=\operatorname{det} A /($ sum of all cofactors). If, in addition, $v(A)=0$, then $K(A)=\left\{q: \pi^{t} q \geq 0\right\}$ for some $\pi>0$.

Theorem 3 (see Murthy, Parthasarathy, and Sriparna [11]). Suppose $A \in \boldsymbol{R}^{m \times n}$. If the matrix game $A$ is completely mixed with $v(A)=0$, then $A \in \boldsymbol{Q}_{0}$ and $K(A)=$ $\left\{q: \pi^{t} q \geq 0\right\}$ for some $\pi>0$. 
Remark 4. It is easy to see that if $A$ is in $\boldsymbol{P}_{1}$ and $\operatorname{det} A=0$, then $A$ is a completely mixed game with $v(A)=0$. However, the converse is not true. This is not true even with an additional assumption that $A$ is a $\boldsymbol{P}_{0}$-matrix. Consider the example

$$
A=\left[\begin{array}{rrr}
0 & -1 & 1 \\
1 & 0 & -1 \\
-1 & 1 & 0
\end{array}\right]
$$

Note that $A$ is a completely mixed game with $A \in \boldsymbol{P}_{0}$ but $A \notin \boldsymbol{P}_{1}$.

Lemma 5. Suppose $A \in \boldsymbol{R}^{n \times n}, n \geq 2$. If $A \in \boldsymbol{E}^{\prime}$, then for every proper subset $\alpha$ of $\bar{n}$ such that $\operatorname{det} A_{\alpha \alpha}=0, A_{\alpha \alpha} \notin \boldsymbol{P}_{1}$.

Proof. Suppose for some proper subset $\alpha, A_{\alpha \alpha} \in \boldsymbol{P}_{1}$ and $\operatorname{det} A_{\alpha \alpha}=0$. Then $A_{\alpha \alpha}$ is a completely mixed game with value 0 . So there exists a positive vector $z_{\alpha}$ such that $A_{\alpha \alpha} z_{\alpha}=0$. Let $q_{\bar{\alpha}}$ be such that $A_{\bar{\alpha} \alpha} z_{\alpha}+q_{\bar{\alpha}}>0$ and $q_{\bar{\alpha}}>0$. Then $z$ is a nontrivial solution of $(q, A)$, where $z^{t}=\left(z_{\alpha}^{t}, 0_{\bar{\alpha}}^{t}\right)$ and $q=\left(0_{\alpha}^{t}, q_{\bar{\alpha}}^{t}\right)$. Since $q$ is a nonzero nonnegative vector, this contradicts the assumption that $A \in \boldsymbol{E}^{\prime}$. This completes the proof.

Theorem 6. Suppose $A \in \boldsymbol{R}^{n \times n}, n \geq 2$. The following statements are equivalent:

(i) $A \in \boldsymbol{P}_{1} \backslash Q$ and $\operatorname{det} A=0$.

(ii) $A \in \boldsymbol{U}$ and $K(A)=\left\{q: \pi^{t} q \geq 0\right\}$ for some positive vector $\pi$.

(iii) $A \in \boldsymbol{E}^{\prime} \cap \boldsymbol{P}_{1}$.

(iv) $A \in \boldsymbol{E}^{\prime} \cap \bar{U}$.

(v) $A \in \boldsymbol{U}$ and $A$ is completely mixed with value zero.

(vi) $A^{t}$ satisfies any one of the above conditions (i)-(v).

(vii) $A^{t}$ satisfies all of the above conditions (i)-(v).

Proof. Cottle and Stone [3] have already proved that (i) implies (ii).

Suppose (ii) holds. Then, as $K(A)$ is a half-space, it is convex and hence $A \in$ $\boldsymbol{Q}_{0} \backslash \boldsymbol{Q}$ (see [5]). So $A \in \boldsymbol{P}_{0}$ (see Theorem 4 of [3]). Since $\boldsymbol{P} \subseteq \boldsymbol{Q}$, $\operatorname{det} A_{\alpha \alpha}=0$ for at least one $\alpha$. If possible, let $\alpha$ be a proper subset of $\bar{n}$ such that $\operatorname{det} A_{\alpha \alpha}=0$. There exists $d_{\alpha} \neq 0$ such that $A_{\alpha \alpha} d_{\alpha}=0$. We may assume that $e_{\alpha}+d_{\alpha}>0$. Let $q_{\alpha}=-A_{\alpha \alpha} e_{\alpha}$. Choose $q_{\bar{\alpha}}$ to be any positive vector satisfying $(a) A_{\bar{\alpha} \alpha} e_{\alpha}+q_{\bar{\alpha}}>0$, (b) $A_{\bar{\alpha} \alpha}\left(e_{\alpha}+d_{\alpha}\right)+q_{\bar{\alpha}}>0$, and $(c) \pi_{\alpha}^{t} q_{\alpha}+\pi_{\bar{\alpha}}^{t} q_{\bar{\alpha}}>0$. Let $q^{t}=\left(q_{\alpha}^{t}, q_{\bar{\alpha}}^{t}\right), z^{t}=\left(z_{\alpha}^{t}, 0_{\bar{\alpha}}^{t}\right)$, and $d^{t}=\left(d_{\alpha}^{t}, 0_{\bar{\alpha}}^{t}\right)$. It is easy to check that $z$ and $z+d$ are two distinct solutions of $(q, A)$. However, since $\pi^{t} q>0, q \in \operatorname{int} K(A)$, this contradicts that $A \in \boldsymbol{U}$. It follows that $\operatorname{det} A=0$ and $A \in \boldsymbol{P}_{1}$. From the corollary of Lemma 1 of [1], $(0, A)$ has at least two solutions. Now it is easy to see that $A \in \boldsymbol{E}^{\prime}$ (also see Danao [4]).

Suppose (iii) holds. Since $A \in \boldsymbol{E}^{\prime}, A \notin \boldsymbol{R}_{0}$. So $A \in \boldsymbol{P}_{1} \backslash \boldsymbol{Q}$. Let $z$ be a nontrivial solution of $(0, A)$. From Lemma 5 , it follows that $z>0$ and hence $A z=0$. From Theorem 1(iii), $A \in U$. As $A \in \boldsymbol{P}_{1}$ and $\operatorname{det} A=0$, all proper principal submatrices of $A$ are in $\boldsymbol{P}$ and hence in $\boldsymbol{U}$. Hence $A \in \overline{\boldsymbol{U}}$.

Suppose (iv) holds. Since $A \in \boldsymbol{E}^{\prime}$ and $n \geq 2$, all diagonal entries of $A$ are positive (if $a_{11}<0$, then $(1,0, \ldots, 0)^{t}$ is a nontrivial solution of $(q, A)$ for some nonzero nonnegative $q$ ). Now, if for some $\alpha$ all proper principal submatrices of $A_{\alpha \alpha}$ are all $\boldsymbol{P}$-matrices and $\operatorname{det} A_{\alpha \alpha}<0$, then $\left(A_{\alpha \alpha}\right)^{-1}$ is an $\boldsymbol{N}$-matrix and hence a $\boldsymbol{Q}_{0}$-matrix (if $A_{\alpha \alpha} \leq 0$, then obviously $\left(A_{\alpha \alpha}\right)^{-1} \in \boldsymbol{Q}_{0}$, and if $A_{\alpha \alpha} \not \leq 0$, then from Theorem 6.6.4 of [2] $\left.\left(A_{\alpha \alpha}\right)^{-1} \in \boldsymbol{Q}\right)$. Since $\boldsymbol{U} \cap \boldsymbol{Q}_{0} \subseteq \boldsymbol{P}_{0}$, this would contradict the hypothesis. So $\operatorname{det} A_{\alpha \alpha} \geq 0$. From Lemma 5, it follows that $\operatorname{det} A_{\alpha \alpha}>0$ for all proper subsets $\alpha$. Since $A \notin \boldsymbol{R}_{0}$, it follows that $A \in \boldsymbol{P}_{1}$, $\operatorname{det} A=0$, and, from Remark 4, $A$ is completely mixed. 
Suppose (v) holds. From Theorem $2, \operatorname{det} A=0$. From Theorem $3, A \in \boldsymbol{Q}_{0}$ and $K(A)=\left\{q: \pi^{t} q \geq 0\right\}$ for some positive vector $\pi$. Since $A \in \boldsymbol{U} \cap \boldsymbol{Q}_{0}, A \in \boldsymbol{P}_{0}$. Suppose $\operatorname{det} A_{\alpha \alpha}=0$ for some $\alpha$ with $|\alpha|<n$. Then there exists $d_{\alpha} \neq 0$ such that $A_{\alpha \alpha} d_{\alpha}=0$. Let $q_{\alpha}=-A_{\alpha \alpha} e_{\alpha}$. Then, for $q_{\bar{\alpha}}$ positive and sufficiently large, we observe $q \in$ int $K(A)$ and $(q, A)$ has two solutions, that is, $\left(e_{\alpha}^{t}, 0_{\bar{\alpha}}^{t}\right)$ and $\left(e_{\alpha}^{t}+\lambda d_{\alpha}^{t}, 0_{\bar{\alpha}}^{t}\right)$ for some $\lambda$ sufficiently small but positive. This contradicts that $A \in \boldsymbol{U}$, and thus all proper principal minors of $A$ are positive. Since $K(A)$ is a half-space, $A \notin \boldsymbol{Q}$.

To complete the rest of the proof, we will show that (i) holds if and only if (i) holds for $A^{t}$. It suffices to show that (i) implies $A^{t} \notin \boldsymbol{Q}$. Suppose (i) holds. From the proof of Lemma 5 it follows that there exists $z>0$ such that $A z=0$. Then $z^{t} A^{t}=0$. Note that this implies $S(q, A)=\emptyset$ for all $q<0$. Hence $A \notin \boldsymbol{Q}$.

3. Block property. The study of stability of solution sets in LCPs leads to the class of Lipschitzian matrices. A matrix $A \in \boldsymbol{R}^{n \times n}$ is said to be Lipschitzian if there exists a real number $\lambda>0$ such that for every $p, q \in K(A)$ and every $z \in S(q, A)$ there exists an $x \in S(p, A)$ such that $\|x-z\| \leq \lambda\|p-q\|$. Mangasarian and Shiau [8] showed that $\boldsymbol{P}$-matrices are Lipschitzian. Gowda [6] showed that if $A$ is Lipschitzian and $(q, A)$ has a unique solution for some nondegenerate $q$ ( $q$ is said to be nondegenerate if $z+A z+q>0$ for all $z \in S(q, A)$ ), then $A$ is in $\boldsymbol{P}$. Later, Murthy, Parthasarathy, and Sabatini [9] showed that a $\boldsymbol{Q}$-matrix is Lipschitzian if it is a $\boldsymbol{P}$-matrix. Stone [15] showed that Lipschitzian matrices are nondegenerate $\boldsymbol{I N} \boldsymbol{S}$-matrices and conjectured that the converse is also true. Murthy, Parthasarathy, and Sriparna [12] introduced the block property and showed that Lipschitzian as well as nondegenerate IN $\boldsymbol{S}$-matrices satisfy the block property; they further conjectured that if a matrix has block property, then it must be Lipschitzian. In this article, we modify the definition of block property and show that the revised property is also a necessary condition for both Lipschitzian and nondegenerate $\boldsymbol{I N S}$-matrices. Furthermore, we show that if a matrix $A$ has the revised block property and $(q, A)$ has a unique solution for some nondegenerate $q$, then $A$ is Lipschitzian.

The main significance of block property (as well as the revised one) is that it can be checked in finite number of steps by examining the sign structures of all PPTs. It must be mentioned here that no finite characterization is known for checking whether a given matrix is a Lipschitzian matrix or not. Thus, if the conjecture is true, one would have a finite condition for checking the Lipschitzian property.

Definition 7. Say that $A \in \boldsymbol{R}^{n \times n}$ has block property $\left(B^{\star}\right)$ if for every PPT $M$ of $A$ there exists a partition $\alpha_{1}, \alpha_{2}, \ldots, \alpha_{k}$ of $\{1,2, \ldots, n\}$ such that the following hold:

(i) $M_{\alpha_{i} \alpha_{i}}$, are negative $\boldsymbol{N}$-matrices for $i=1,2, \ldots, k-1, M_{\alpha_{i} \alpha_{j}}=0$ for $i \neq j$, $i, j=1,2, \ldots, k-1$, and the diagonal entries of $M_{\alpha_{k} \alpha_{k}}$ are positive.

(ii) For any index sets $\alpha$ and $\beta$ such that $\alpha \Delta \beta=\{i\}$ for some $i$, $\operatorname{det} M_{\alpha \alpha} \operatorname{det} M_{\beta \beta}>$ 0 provided $\left\{x \in \boldsymbol{R}_{+}^{n-1}:\left[-M_{. \alpha} I_{. \bar{\alpha}}\right]_{.(i)} x>0\right\} \neq \emptyset$.

Theorem 8. Suppose $A \in \boldsymbol{R}^{n \times n}$ is a nondegenerate INS-matrix. Then $A$ has property $\left(B^{\star}\right)$.

Proof. In [12] it was shown that $A$ satisfies condition (i) of Definition 7. From Theorem 6.6.20 of [2], it follows that $M$ does not have any reflecting facets (see also Definition 6.2.10 and Definition 6.6.14 of [2]). From this, the second condition of the definition follows.

Corollary 9. Suppose $A \in \boldsymbol{R}^{n \times n}$ is a Lipschitzian matrix. Then $A$ has prop$\operatorname{erty}\left(B^{\star}\right)$. 
Proof. The proof follows from the fact that Lipschitzian matrices are nondegenerate $\boldsymbol{I N} \boldsymbol{S}$-matrices.

Theorem 10. Suppose $A \in \boldsymbol{R}^{n \times n} \cap \boldsymbol{E}_{0}$. Assume that $A$ has property $\left(B^{\star}\right)$. Then $A \in \boldsymbol{P}$.

Proof. We will prove this by induction. Since $A \in \boldsymbol{E}_{0}, a_{i i}>0$ for all $i$. Assume that $A_{\alpha \alpha} \in \boldsymbol{P}$ for all $\alpha$ such that $|\alpha|=k-1, k \geq 2$. Let $\beta$ be any arbitrary index set such that $|\beta|=k$. Suppose $\operatorname{det} A_{\beta \beta}<0$. Then the inverse of $A_{\beta \beta}$ is an $\boldsymbol{N}$-matrix. From (i) of property $\left(B^{\star}\right),\left(A_{\beta \beta}\right)^{-1} \leq 0$ and all its diagonal entries are negative. This would imply that $v\left(\left(A_{\beta \beta}\right)^{-1}\right)<0$ and hence $v\left(A_{\beta \beta}\right)<0$. However, this is not possible, as $A \in \boldsymbol{E}_{0}$ (see Definition 3.9.4 and Corollary 3.9.7 of [2]).

Theorem 11. Suppose $A \in \boldsymbol{R}^{n \times n}$ has property $\left(B^{\star}\right)$. Assume that $(q, A)$ has a unique solution for some nondegenerate $q$. Then $A \in \boldsymbol{P}$.

Proof. We may assume without loss of generality that $q>0$. If $(p, A)$ has a unique solution for every positive vector $p$, then $A \in \boldsymbol{E}_{0}$ and hence $A \in \boldsymbol{P}$. Suppose, to the contrary, that $(p, A)$ has a nontrivial solution for some $p>0$. This means there exists a facet in the positive orthant and an open ball $B$ of positive vectors such that for every $q^{\prime} \in B \cap H^{+},\left(q^{\prime}, A\right)$ has a unique solution, and for every $q^{\prime} \in B \cap H^{-}$, $\left(q^{\prime}, A\right)$ has at least two solutions, where $H^{+}$and $H^{-}$are the half-spaces containing the facets. Let $\alpha$ be the index set corresponding to the facet. Then $\alpha \neq \emptyset$. Since the facet intersects the nonnegative orthant in the interior, the system defined in condition (ii) of Definition 7 is nonempty. Condition (ii) of the definition implies for $i \notin \alpha,-A_{. i}$ and $I_{. i}$ are on the opposing sides of the facet in question. But then it would mean that $\left(q^{\prime}, A\right)$ has at least two solutions for some $q^{\prime} \in B \cap H^{+}$-a contradiction. It follows that $(p, A)$ has a unique solution for every positive vector $p$. Hence $A \in \boldsymbol{E}_{0}$ and the rest follows from Theorem 10.

From the above we have a characterization of $\boldsymbol{P}$-matrices (see [2] for characterizations of $\boldsymbol{P}$-matrices), namely, $A \in \boldsymbol{P}$ if and only if $A$ has property $\left(B^{\star}\right)$ and $(q, A)$ has a unique solution for some nondegenerate $q$.

Acknowledgments. The authors wish to thank Prof. O. J. Vrieze for some useful discussions and for inviting the first two authors to visit the Department of Mathematics, University of Maastricht. The first author offers special thanks to Prof. K. G. Murty, University of Michigan at Ann Arbor, for partially supporting his foreign tour.

\section{REFERENCES}

[1] M. Aganagic and R. W. Cottle, A note on Q-matrices, Math. Programming, 16 (1979), pp. $347-377$.

[2] R. W. Cottle, J. S. Pang, and R. E. Stone, The Linear Complementarity Problem, Academic Press, Boston, 1992.

[3] R. W. Cottle And R. E. Stone, On the uniqueness of solutions to linear complementarity problems, Math. Programming, 27 (1983), pp. 191-231.

[4] R. A. DanaO, A note on $E^{\prime}$-matrices, Linear Algebra Appl., 259 (1997), pp. 299-305.

[5] B. C. EAves, The linear complementarity problem, Management Sci., 17 (1971), pp. 612-634.

[6] M. S. GowDA, On the continuity of the solution map in linear complementarity problems, SIAM J. Optim., 2 (1992), pp. 619-634.

[7] I. Kaplansky, A contribution to von Neumann's theory of games, Ann. of Math. (2), 46 (1945), pp. $474-479$.

[8] O. L. Mangasarian and T.-H. Shiau, Lipschitz continuity of solutions of linear inequalities, programs and complementarity problems, SIAM J. Control Optim., 25 (1987), pp. 583-595.

[9] G. S. R. Murthy, T. Parthasarathy, and M. Sabatini, Lipschitzian Q-matrices are Pmatrices, Math. Programming, 74 (1996), pp. 55-58. 
[10] G. S. R. Murthy, T. Parthasarathy, and B. Sriparna, Constructive characterization of Lipschitzian Q0-matrices, Linear Algebra Appl., 252 (1997), pp. 323-337.

[11] G. S. R. Murthy, T. Parthasarathy, and B. Sriparna, Linear complementarity problems in static and dynamic games, Ann. Dynamic Games, to appear.

[12] G. S. R. Murthy, T. Parthasarathy, and B. Sriparna, Some recent results on the linear complementarity problem, SIAM J. Matrix Anal. Appl., 19 (1998), pp. 898-905.

[13] K. G. Murty, Linear Complementarity, Linear and Nonlinear Programming, HeldermannVerlag, Berlin, 1988.

[14] T. D. Parsons, Applications of principal pivoting, in Proceeding of the Princeton Symposium on Mathematical Programming, H. W. Kuhn, ed., Princeton University Press, Princeton, NJ, 1970, pp. 561-581.

[15] R. E. Stone, Lipschitzian matrices are nondegenerate INS-matrices, in Complementarity and Variational Problems, C. Ferris and J. S. Pang, eds., SIAM, Philadelphia, PA, 1997, pp. $440-451$. 